\title{
Modelling and Verifying Communication Failure of Hybrid Systems in HCSP
}

\author{
Wang, Shuling ; Nielson, Flemming; Nielson, Hanne Riis; Zhan, Naijun
}

Published in:

Computer Journal

Link to article, DOI:

10.1093/comjnl/bxw084

Publication date:

2016

Document Version

Early version, also known as pre-print

Link back to DTU Orbit

Citation (APA):

Wang, S., Nielson, F., Nielson, H. R., \& Zhan, N. (2016). Modelling and Verifying Communication Failure of Hybrid Systems in HCSP. Computer Journal, 60(8), 1111-1130. https://doi.org/10.1093/comjnl/bxw084

\section{General rights}

Copyright and moral rights for the publications made accessible in the public portal are retained by the authors and/or other copyright owners and it is a condition of accessing publications that users recognise and abide by the legal requirements associated with these rights.

- Users may download and print one copy of any publication from the public portal for the purpose of private study or research.

- You may not further distribute the material or use it for any profit-making activity or commercial gain

- You may freely distribute the URL identifying the publication in the public portal 


\title{
Modification of SOL profiles and fluctuations with line-average density and divertor flux expansion in TCV
}



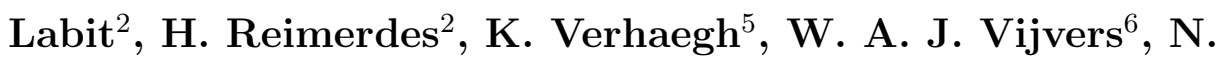 \\ Walkden $^{4}$, S. Costea ${ }^{7}$, J. Kovacic ${ }^{8}$, C. Ionita ${ }^{7}$, V. Naulin ${ }^{9}$, A. H. \\ Nielsen $^{9}$, J. J. Rasmussen ${ }^{9}$, B. Schneider ${ }^{7}$, R. Schrittwieser ${ }^{7}$, \\ M. Spolaore ${ }^{1}$, D. Carralero ${ }^{10}$, J. Madsen ${ }^{9}$, B. Lipschultz ${ }^{5}$, F. \\ Militello $^{4}$ the TCV Team and the EUROfusion MST1 Team $\ddagger$ \\ ${ }^{1}$ Consorzio RFX (CNR, ENEA, INFN, Universitá di Padova, Acciaierie Venete SpA), \\ Corso Stati Uniti 4, 35127 Padova, Italy ${ }^{2}$ Swiss Plasma Center (SPC), EPFL \\ Lausanne, Switzerland, ${ }^{3}$ UCSD, La Jolla, USA, ${ }^{4}$ CCFE, Culham Science Centre, \\ Abingdon OX14 3DB, UK, ${ }^{5}$ York Plasma Institute, University of York, York, UK, \\ ${ }^{6}$ DIFFER - Dutch Institute for Fundamental Energy Research, De Zaale 20, 5612 AJ \\ Eindhoven, the Netherlands, ${ }^{7}$ Institute for Ion Physics and Applied Physics, \\ Innsbruck, Austria, ${ }^{8}$ Jozef Stefan Institute, Ljubljana, ${ }^{9}$ DTU, Copenhagen, \\ Denmark, ${ }^{10}$ Max-Plank-Institut fur Plasmaphysik, Garching, Germany \\ E-mail: nicola.vianello@igi.cnr.it
}

\begin{abstract}
A set of Ohmic density ramp experiments addressing the role of parallel connection length in modifying Scrape Off Layer (SOL) properties has been performed on the TCV tokamak. The parallel connection length has been modified by varying the poloidal flux expansion $f_{x}$. It will be shown that this modification does not influence neither the detachment density threshold, nor the development of a flat Scrape Off Layer (SOL) density profile which instead depends strongly on the increase of the core line average density. The modification of the SOL upstream profile, with the appearance of what is generally called a density shoulder, has been related to the properties of filamentary blobs. Blob size increases with density, without any dependence pn the parallel connection length both in the near and far SOL. The increase of the density decay length, corresponding to a profile flattening, has been related to the variation of the divertor normalized collisionality $\Lambda_{d i v}$ [1, 2], showing that in TCV the increase of $\Lambda_{d i v}$ is not sufficient to guarantee the SOL upstream profile flattening.
\end{abstract}

PACS numbers: 52.25.Xz, 52.35.Ra, 52.35.We, 52.70.Ds

$\ddagger$ See the author list of Overview of progress in European Medium Sized Tokamaks towards an integrated plasma-edge/wall solution by H. Meyer et al., to be published in Nuclear Fusion Special issue: overview and summary reports from the 26th Fusion Energy Conference (Kyoto, Japan, 17-22 October 2016) 
To be Submitted to: Nucl. Fusion 


\section{Introduction}

Plasma Wall Interaction (PWI) is a subject of intense study in the context of fusion energy research for the understanding of heat load, sputtering levels, lifetime of the Plasma Facing Components (PFC) and amount of tritium retention in wall structures. Plasma flux towards the first wall or towards the divertor plate is determined by the competition between losses parallel and perpendicular to the magnetic field in the Scrape Off Layer (SOL). The understanding of these transport mechanisms in different plasma regimes is essential, and has already had clear impact in future machine design [3]. All operational scenarios expected in future machines like ITER or DEMO require high values of Greenwald fraction and partial or complete detachment. In high density scenarios, even without reaching detachment conditions, the L-Mode SOL exhibits a modification of the density profile, with a general broadening (also named profile shoulder) as observed in a variety of different devices [4, 5, 6, 7]. While this is a well established behavior for the L-Mode regime, H-Mode behavior is less clear, even though preliminary results suggest that a similar mechanism occurs also in high power operation $[8,9,10]$.

Since the early observations SOL profile broadening [4], its happening has been attributed to an enhancement of cross field advective transport. In addressing this topic it has to be considered that SOL transport is dominated by the radial outward propagation of intermittent convective structures, named filaments or blobs [11], which convey particles and energy towards the first wall and can be responsible for up to 50 $\%$ of the fluctuation induced radial transport [11].

Basic models for filaments [12] describe their propagation as the result of a combination of plasma polarization, due to the $\nabla B$ drift, and parallel closure. Originally the proposed scheme, dubbed the sheath connected regime, considered the filament circuit closed through the divertor plates with the main potential drop at the sheath entrance. This model has been refined, with different closures, including the possibility for the filament to be electrical disconnected from the divertor plates which leads the ion cross-field inertia providing the closure mechanism for the blob, termed the inertial regime [13, 14, 15, 16]. Furthermore it has been suggested that electrical connection could be also established through the plasma background [17]. Experimental verification of existence of these two regimes has been obtained for example in the simple magnetized plasma TORPEX [18].

An important consequence is that filaments in the different regimes exhibit different velocity scaling properties with respect to filament size [1]. One of the theoretical frameworks describing this transition has been proposed in [1], where the filament parameter space has been described in the $\Lambda-\Theta$ plane, where:

$$
\Lambda=\frac{L_{\|}}{c_{s}} \nu_{e i} \frac{\Omega_{i}}{\Omega_{e}} \quad \Theta=\left(\frac{\delta_{b} R^{1 / 5}}{L_{\|}^{2 / 5} \rho_{s}^{4 / 5}}\right)^{5 / 2}
$$

with $L_{\|}$the parallel connection length, $c_{s}$ the ion sound speed, $\nu_{e i}$ the electron-ion 
collision frequency, $\Omega_{i}, \Omega_{e}$ the ion and electron gyrofrequency respectively, $\delta_{b}$ the blob size and $\rho_{s}$ the ion sound gyroradius, and $\mathrm{R}$ is the major radius. The two quantities represent respectively a normalized collisionality and a normalized blob-size: at constant blob-size, the increase of collisionality implies the transition from sheath-limited to inertial regime, which is physically associated to the electrical disconnection of the filaments from the target.

Recently the development of a SOL density profile shoulder observed in L-Mode high density discharges in AUG and JET has been associated with the aforementioned transition from sheath-limited to inertial regime [2]. Indeed in the inertial regime, velocity scales as the square root of blob size $\delta_{b}, v_{r} \sim \sqrt{\delta_{b}}$ and larger blobs with larger velocity has been advocated as the cause of increasing convective radial transport in the higher density regime. More recent results from JET [19] revealed that the shoulder tends to disappear whenever the strike point is moved from the horizontal to the vertical target and that nitrogen seeded discharges exhibit a flatter profile only at very large values of $\Lambda_{d i v}$, defined as the parameter $\Lambda$ introduced in Equation (1) computed with the typical values at the separatrix, in the mentioned case close to the separatrix. Furthermore the development of a SOL density shoulder has been observed to strongly depend on the plasma current $\mathrm{I}_{p}[5,20,21]$ : flat profiles observed at certain levels of the core average density disappear whenever the plasma current is increased. Consequently it is clear that a unified picture of the process is presently lacking although the enhancement of convective filamentary transport is a generally accepted result.

Within this framework, intense experimental activity has been carried out in the TCV tokamak [22], exploiting the flexibility of this device to test shoulder formation against variation of magnetic geometry. Shoulder formation and blob properties have been investigated in a series of L-Mode density ramps performed at different poloidal flux expansion values, in order to address the role of parallel connection length in the process of SOL profile flattening. This paper will show that SOL shoulder formation does not depend on the value of parallel connection length within the achieved variation of $\mathrm{L}_{\|}$. As far as fluctuations are concerned, we will show that the density decay length in the far SOL depends on the blob size consistently with other devices, whereas no relation is found in the near SOL. Blob size is shown to strongly depend on density with no dependence on the parallel connection length. The dependence of the density decay length on the divertor collisionality $\Lambda_{d i v}$ is weaker than in ASDEX or JET and high level of $\Lambda_{d i v}$ is found to be a necessary but not sufficient conditions in order to obtain a flatter density profile.

The paper is organized as follows: in section 2 the experimental setup and available diagnostics will be presented; in section 3 the main experiment, consisting of Ohmic discharges at different flux expansion will be described, focusing on the behavior of target profiles in section 4 and upstream profiles in section 5 and providing differences in terms of filament properties in section 6 . In section 7 we provide a unified picture of the observed behavior within the framework proposed in [1] and finally, in section 8 , we draw the conclusions. 


\section{Experimental setup}

The Tokamak à Configuration Variable (TCV) is a medium size tokamak located at the Swiss Plasma Center, Switzerland. It is a conventional aspect ratio tokamak (major and minor radii $R=0.88 \mathrm{~m}$ and $a=0.25 \mathrm{~m}$ respectively) with a highly elongated vacuum vessel and a completely open divertor. TCV features 16 independently powered poloidal field coils, resulting in unique shaping capabilities, with the ability to accommodate highly elongated plasmas (up to 2.8 ) and triangularity in the range $-0.7 \leq \delta \leq 1$. The near-complete coverage of TCV surfaces with graphite tiles allows extreme flexibility in power load deposition, making TCV an ideal test-bed for the study of different magnetic configurations and divertor geometries.

In recent years great efforts have been devoted in TCV to increase the diagnostic equipment for divertor studies. A new 32-chords divertor spectroscopy system (DSS) [23] has been installed for extracting information on recombination and electron temperature from Balmer series spectra. This information can be combined with radiation measurements estimated from Bolometry [24] and absolute extreme ultraviolet (AXUV) bolometric camera sightlines [25]. An array of wall-mounted Langmuir probes (LPs) [26] covers the inner and outer wall as well as the floor. The cylindrical tips have diameters of $4 \mathrm{~mm}$ and are embedded into the tiles except at the floor where they have a dome-shaped head protruding from the tile shadow by $1 \mathrm{~mm}$. Among the available 114 LPs, only 48 can currently be used simultaneously. They have been operated in swept mode to obtain density and temperature profiles at the wall. The set of LPs has been extended by the installation of a fast reciprocating probe [27]. The probe head, described in detail elsewhere [27], is equipped with 10 graphite electrodes $1.5 \mathrm{~mm}$ in diameter. The electrodes are arranged in such a way to provide a double probe for density and temperature profiles, two Mach probes for parallel flow investigations, a pin for fast ion saturation current measurements with the remaining probes collecting floating potential in order to infer radial and poloidal electric field fluctuations from the local floating potential gradient. The radial separation between the floating potential pins is $1.57 \mathrm{~mm}$ whereas the poloidal separation between the pins are $4 \mathrm{~mm}$ and 10 $\mathrm{mm}$ respectively. The fast movement of the probe head is $20 \mathrm{~cm}$ which is reached within $90 \mathrm{~ms}$ with a maximum speed of $2.2 \mathrm{~m} / \mathrm{s}$. The acquisition frequency of the system is $2.5 \mathrm{MHz}$ with a bandwidth for ion saturation current and floating potential of approximately $5 \mathrm{MHz}$. The Line of Sights (LOS) of bolometry and the location of the Langmuir probes and of the reciprocating manipulator used throughout the paper are shown in Figure 1 to provide an idea of the spatial resolution of the diagnostic setup. The combined information provide a comprehensive set of measurements suited for the divertor investigation.

As an example of the mechanism we will investigate in the paper, Figure 2 shows upstream density profiles, as obtained by the reciprocating probe in 3 similar L-mode LSN discharges, at three levels of core line-averaged density. The SOL profiles are normalized to the density value at the separatrix and a clear flattening of the profile is 
obtained as the density is increased in accordance with previous TCV observation [6].

\section{Experimental scenarios: flux expansion scan}

The establishment of a flatter SOL profile is basically determined by a modification of the ratio of perpendicular to parallel losses $\Gamma_{\perp} / \Gamma_{\|}$, under the assumption of small variation of the source term in the particle balance equation. Furthermore the effective collisionality $\Lambda$ defined in Equation (1) is directly proportional to $\mathrm{L}_{\|}$, and any variation of the parallel connection length should reflect immediately on $\Lambda$. In addition, the modification of the parallel connection length was demonstrated to influence blob dynamics in the simple magnetized torus TORPEX [28]. Starting from these motivations a set of experiments has been conceived in order to investigate the dependence of the SOL shoulder formation process on the parallel connection length $\mathrm{L}_{\|}$. A series of density ramp discharges were carried out in Ohmically heated L-mode plasmas. The poloidal flux expansion is defined as

$$
f_{x}=\frac{B_{\theta}^{u} B_{\phi}^{t}}{B_{\theta}^{t} B_{\phi}^{u}}
$$

with $u$ and $t$ denoting upstream and target quantities, and $\theta$ and $\phi$ the poloidal and toroidal component respectively, has been varied in between the shots from $f_{x} \approx 2$ to $f_{x} \approx 11$. From the geometrical point of view the increase of the flux expansion causes an increase of the flux tube volume [29] through changes in the the parallel connection length between upstream and the target as well as an increase of the plasma-wetted area. Examples of the configuration variation from smaller to larger flux expansion are shown in Figure 3. As we can observe from panel (a) of Figure 3, the value $\mathrm{L}_{\|}$ from the midplane to the outer target almost doubles throughout the SOL when the flux expansion is increased. The computation of the parallel connection length from the primary X-point to the LFS target, shown in panel (b) of the same Figure 3, reveals that the increase of $\mathrm{L}_{\|}$is even larger in the divertor region with a ratio between larger and smaller $f_{x}$ up to a factor of 3.In the following sections we will provide detailed analysis on the effect of core plasma density ramps on divertor (cfr. section 4) and upstream (cfr section 5) profiles highlighting any differences caused by the modification of the flux expansion.

\section{Divertor modification}

The effect of increasing the flux expansion on target profiles has been previously studied in $[30,31]$. In the present paper we extend these experimental observations through simultaneous modification of the upstream and target plasmas, with a focus on filamentary properties. In Figure 4 we report results from the identical core plasma density ramp performed in two different shots at two different flux expansions. In panels (a) and (b) of Figure 4 the brightness from three bolometer LoS observing the outer divertor leg as a function of line average density are shown. The radiation starts moving 
from the target at lower core density for the larger flux expansion but the maximum of the radiation reaches the chord looking closer to the $\mathrm{X}$-point at the same density value. Panels (c) and (d) of the same Figure 4 show the spatially integrated total ion flux to the outer strike point, still as a function of line average density. The ion flux increases almost linearly for both flux expansion cases, thus diverging from the theoretically predicted square dependence expected in high-recycling regime [32] indicated in the same panels with grey dashed line. At high values of line-averaged density $\left(\left\langle n_{e}\right\rangle \approx 7 \times 10^{19} \mathrm{~m}^{-3}\right)$ both shots exhibit a drop in the total collected ion flux, indicating the typical rollover on target density during density ramps. Actually the ion flux rollover density for both the values of flux expansions (or $\mathrm{L}_{\|}$) remains approximately constant, confirming that increasing flux expansion does not substantially modify rollover density threshold as in the reverse $B_{\phi}$ cases at higher current [31].

Apart from the effect on the total ion current we can provide detailed experimental evidences of the effect on target density profiles shown in Figure 5. Panels (b) and (e) show the LFS target density profiles at two levels of line-averaged density for two values of flux expansion. At lower density (Figure $5(\mathrm{~b})$ ) the profiles obtained at different $\mathrm{f}_{x}$ are almost identical. Increasing the density (Figure 5 (c)) causes a broadening of the profiles for both the values of flux expansion although at larger values of $\mathrm{f}_{x}$ the density value is lower all along the profile.

\section{Upstream profile modification}

At the midplane SOL density profiles are reconstructed using double probe mounted on a multi-pin probe head on the reciprocating manipulator [27]. In Figure 5 (a) and (b) we provide these profiles at two levels of density for the two flux expansions. We observe that the density profiles in the SOL are unaffected by the modification of the flux expansion for both the values of density considered, and the flatter profile observed between $1.025 \lesssim \rho_{\Psi} \lesssim 1.06$ in panel $(\mathrm{d})$ appears as a pure consequence of density increases. In the bottom panels of the same Figure 5, (c) and (f), we compare the values of $\Lambda_{\text {div }}$ computed according to Equation (1) with density and temperature taken at the LFS target and $\mathrm{L}_{\|}$estimated as the parallel connection length from the primary $\mathrm{X}$-point to the LFS target. A strong variation in the $\Lambda_{\text {div }}$ profiles is observed between the two flux expansion cases and the profile lies well above the value of $\Lambda_{\text {div }}=1$ identified in [2] as the threshold for the appearance of shoulder in the SOL profile. But even though $\Lambda_{\text {div }}$ reaches very high values all along the SOL width, and differences exist between the two flux expansion cases, no evidence of a very broad and flat density profile is observed in neither of the two cases and profile differences are observed only as a function of density.

The SOL density profile dependence is confirmed on a statistical basis in Figure 6

where the density decay length $\lambda_{n}=\left(\frac{\left|\nabla n_{e}\right|}{n_{e}}\right)^{-1}$ is shown for both the near and far SOL (respectively defined as being between $1 \leq \rho_{\Psi} \leq 1.025$ and $1.025<\rho_{\Psi} \leq 1.06$ ) as a function of flux expansion, subdividing the data in ranges of core line average density 
(panel (a) and (b) respectively). Clearly in both the near and far SOL the decay length does not exhibit any clear trend with flux expansion, confirming that upstream profiles are unaffected by the increase of $\mathrm{L}_{\|}$. The same exercise has been repeated on the same database by computing the dependence of $\lambda_{n}$ on line average density, still distinguishing the near and far SOL. The results are shown in panel (c) and (d) of Figure 6. The near SOL decay length clearly increases with density, without any clear distinction within the various ranges of flux expansion: the maximum near SOL $\lambda_{n}$ value reached around $30-40 \mathrm{~mm}$ which is close to the average value observed in the far SOL (cfr. Figure 6 (d)). In the latter region the trend is less clear and the profiles seem to be rather constant with density. It is worth mentioning that these results confirm on a stronger statistical basis the observations reported in [6]. Indeed by considering Fig. 3 of reference [6] we observe that also in those experiments the process of shoulder formation was most strongly correlated with the increasing of the density decay length in the near SOL region (marked in the aforementioned Fig. 3 from 0 (separatrix) to 0.25 ) whereas the region in the far SOL (from 0.5 to 1 in their system of reference) exhibits a less pronounced variation of the $\lambda_{n}$ with the increase of the line-averaged density.

\section{Filaments characteristics as a function of density and $L_{\|}$}

Analysis was performed on fluctuations in the signal collected using the fast probe in order to investigate modification of filamentary structures. The analysis has been performed using standard conditional average sampling technique (CAS) as described, for example, in [11]. We have used subsamples of 3-4 ms with a peak amplitude for blob identification larger than 2.5 times the rms value. The chosen length gives sufficiently robust statistics on filaments with the number of detected structure of the order of $\sim 30-70$, depending on the different conditions. The blob size is computed from the FWHM size, $\tau_{b}$, of the conditionally sampled ion saturation current $\mathrm{I}_{s a t}$, multiplied by $v_{\perp}=\sqrt{v_{r}^{2}+v_{p}^{2}}$, according to :

$$
\delta_{b}=\tau_{b} v_{\perp}
$$

where $v_{r}$ and $v_{p}$ are the radial and poloidal components of the velocity respectively. Given the configuration of the probe head, the radial velocity fluctuations have been estimated from the poloidal electric field using the floating potential gradient. Concerning the poloidal propagation, the small radial separation of the pins and the lack of information on the fast temperature gradient prevent us from using $\mathbf{E} \times \mathbf{B}$ estimate. Instead we use the cross-correlation between the conditionally sampled floating potentials at different poloidal positions, similarly to what is done for ion saturation currents in [7]. The results of this analysis for 4 cases are shown in Figure 7: 3 of these cases were obtained at the same core density $\left(\approx 5 \times 10^{19} \mathrm{~m}^{-3}\right)$ but different flux expansion $\left(f_{x}=2.1,4.3,11.9\right.$ respectively), whereas the last one is obtained at higher core density in a small flux expansion case. The asymmetrical shape of the ion saturation current structure can be recognized in all cases, and this is consistent 
with the shape already reported for example in [33]. The increase of flux expansion, and consequently of the parallel connection length, does not change significantly the filament properties: indeed the blob size, averaged in the region $1.025 \leq \rho_{\Psi} \leq 1.06$ is rather constant as a function of $\Lambda_{d i v}$ (estimated as an average over the same region) for three shots within the $f_{x}$ scan, as shown in Figure 7 panel (e). On the other hand, increasing the density significantly increases the filament size (orange point in Figure 7 (e)), without substantial modification of its radial velocity (see panel (b) of the same figure): this increase is achieved at the lower values of $\Lambda_{\text {div }}$ explored within the scan shown in Figure 7. This definitively proves that $\Lambda_{\text {div }}$ is not a sufficient parameter to guarantee filament size increase or profile flattening whereas the density seems to play a major role.

The dependence of the filament size on the level of core density and the lack of relation with the flux expansion are also confirmed on a statistical basis. Indeed, blob size is investigated as a function of core density, subdividing in ranges of flux expansion, as well as a function of flux expansion, dividing in ranges of density. The results are shown in Figure 8 for both the near and far SOL, still defined as the region before and after 1.025 in normalized poloidal flux. The filament size does not exhibit any dependence on flux expansion, and thus on the parallel connection length and this is true in both regions of the SOL as seen from panel (a) and (b). The size increases instead as a function of line average density in particular in the near SOL (panel (c) of the same Figure 8), and this is true for all flux expansions ranges. This is in line with previous observations [34] which demonstrated the tendency of the density fluctuations to increase with line average density. The trend in the far SOL is less clear with a slight tendency towards an increase for very high values of density (panel (d)). Probe measurements do not allow for estimates of blob size independently of their velocity. Given the observed dependency on density, we have analyzed all the quantities defining the blob size, given in Equation (3), as a function of density both in the near and far SOL. The results are shown in Figure 9, still distinguishing in classes of flux expansion. The increase of blob size in the far SOL is given mainly by the increase of $\tau_{b}$ which more than doubles from smaller to larger density. The poloidal velocity is fairly constant in this region, whereas the radial velocity exhibits a moderate reduction (of the order of $30-35 \%$ from the larger to the smaller value in density) The order of magnitude of the radial velocity between 2 and $3 \mathrm{~km} / \mathrm{s}$ is of the order of $5 \times 10^{-2}$ of the local ion sound speed $\mathrm{c}_{s}$, slightly higher than what reported in [35], even though in this publication a large scatter in the estimates of $\mathrm{v}_{r}$ is shown. It is worth mentioning that measurements at the wall performed both at JET [36] and in the past on TCV [6] reveal an opposite tendency of the shape $\tau_{b}$ with the density. In the near SOL, the radial velocity of the filaments is fairly constant or at most slightly decreases with density with increments of $\tau_{b}$ (Figure 9 (a)), similar to the Far SOL region. This increment is partially compensated by the decrease in amplitude of the poloidal velocity (Figure 9 (c)). The fact that the poloidal velocity varies with density in the SOL has been previously observed as for example in C-Mod [37]. 


\subsection{Fast camera measurements}

Filamentary properties and dynamics have also been investigated with fast cameras. Measurements have been made using a fast framing camera, mounted with a tangential view into the TCV vessel at the midplane, to complement the analysis of filamentary structures made by the midplane probe. The camera model was a Photron SA4 camera operated at a $100 \mathrm{kHz}$ frame rate. The camera views almost an entire half of the TCV torus. However, due to shadowing by the viewing port window, the outboard side of the plasma is obscured. This means that obtaining information on the radial geometry and/or motion of filamentary structure is not possible with the camera view. However, filaments, being field aligned, wrap around the plasma torus and pass close to the port window, allowing for high spatial resolution imaging of their poloidal geometry and motion. In this sense the measurements made on the camera are not directly comparable to the probe measurements, but are complementary.

To extract filamentary structures from the data obtained by the fast camera, a background subtraction is applied. The pixel-wise minimum of the 19 preceding frames is subtracted from the current frame. The resulting image samples only the fast-varying component of the movie, but also displays a significant noise component. To reduce the impact of this salt-noise (positive definite random noise) a Gaussian blur is applied with $\sigma=1$. The structures analysed in the subsequent analysis are much larger than this blur radius and as such the blur has no impact on the results drawn here. This has been confirmed by varying $\sigma$, which did not vary the results presented herein, but successfully mitigated the effect of the noise. Figure 10 shows the image processing steps outlined on an example frame from the fast camera.

To extract the structure of a typical filament from the processed movies, a crosscorrelation technique has been applied. This proceeds by calculating the correlation of the time-series from a selected pixel in the image with all other pixels in the image frame. This results in a 2D image of the cross-correlation which can be interpreted as the structure of a typical fluctuation that produces a signal on the selected pixel. In other words, when the selected pixel is lit by a fluctuation in the light emission from the plasma, this technique extracts the most probable spatial structure of such a fluctuation as observed by the camera. Furthermore a time-delay, $\tau$, can be introduced into the cross-correlation which then provides a measurement of the structure of a fluctuation $\tau$ before/after it appears on the selected pixel. This technique can thus be used to generate images of the structure and flow of a typical fluctuation that lights up the selected pixel. To ensure that the measurements made are statistically significant, the cross-correlation has been calculated over a series of 2500 frames (25 ms in time) from plasmas in both a scan in flux-expansion and a scan in density. Figure 11 (left most plot) shows the result of this cross-correlation measurement applied to the medium flux-expansion case. The structure of the cross-correlation (shown in color) is a band extending across the image which follows the path of the toroidal magnetic field. It is relatively localised in the vertical (approximately poloidal) direction and can therefore be associated with the 
structure of a filament. The flow pattern derived from the time-delayed cross-correlation shows a motion of the filament vertically in the camera view, which corresponds to a poloidal rotation of the filament.

To compare the filament structure and motion across scans in flux-expansion and density a vertical cut of the cross-correlation image has been taken at $\tau=-10,0$ and 10 $\mu$ s respectively. The position of this cut is indicated by the blue line in figure 11, left. Comparing the shape of the cross-correlation along this line at $\tau=0$ (red lines in figure 11 centre and right plots) shows that neither flux-expansion or varying electron density has a detectable impact on the poloidal width of the filaments. The poloidal velocity can be inferred by the difference in the location of the peaks in the correlation at $\tau=-10$ and $\tau=10 \mu \mathrm{s}$. In the case of varying flux-expansion, no significant change in the poloidal velocity is observed across the scan, indicating that flux-expansion does not impact the poloidal features of filaments in the plasmas studied here. By contrast, increasing the line-averaged density leads to a reduction in the poloidal velocity (characterised by a shifting of the blue and black curves towards one another) by approximately a factor of 2 . These results therefore suggest that increasing the line-averaged density slows the filament poloidal rotation velocity, consistent with the observation obtained from the probe in the near SOL region shown in Figure 9 (c) in the same flux expansion range (the lower one).

\section{7. $\Lambda_{\text {div }}$ and $\Theta$ scaling}

As mentioned above, it has been suggested that the flattening of the SOL density profile and the modification of filamentary properties are intimately connected: the behavior of these quantities has been recently reconciled through the scaling properties with respect to the divertor normalized collisionality [2]. To test whether this scaling is recovered also in the TCV experimental data we have computed the density decay length in the far and near SOL as a function of $\Lambda_{d i v}$ and $\Theta$ : the latter has been estimated using the local divertor parameters (density, temperature and $\mathrm{L}_{\|}$). The results of this analysis are shown in Figure 12 where the color code indicates the parallel connection length. Clearly, the density decay length in the near SOL does not depend neither on $\Theta$ nor $\Lambda_{\text {div }}$ (panel (a) and (b) respectively), and neither on the parallel connection length. Despite the large scatter encountered in the data, we can observe instead that $\lambda_{n}$ in the far SOL tends to increase whenever $\Theta$ and $\Lambda$ are larger then 1, although none of the two conditions are sufficient to ensure SOL profile flattening. In order to link the experimental observation with the theoretical framework described in [1], we consider in Figure 13 (a) the $\Lambda-\Theta$ plot for the points collected in the far SOL, where density decay length and filament behavior are found to be connected. According to the definitions given in [1] the orange and blue points should be in the so called resistive balloning and resistive $X$-point regimes, where the blobs are electrically disconnected from the target. The size-velocity scaling properties in these two cases are expected to be different [38]. In the same Figure 13, panel (b), we show the same $\lambda_{n}$ vs $\Lambda_{\text {div }}$ plot classifying the points 
according to the scheme shown in panel (a) of the same figure. Clearly whenever the blobs are in the sheath-connected regime (SC), i.e. electrically connected to the plates, the upstream profile exhibits a steep gradient without shoulder. The larger values of $\lambda_{n}$ are obtained whenever the blob is in the resistive $X$-point (RX) regime with few cases in the Resistive Balloning $(\mathrm{RB})$ regime. However, whenever $\Lambda_{\text {div }}$ is increased even further, a regime without any clear tendency towards a flat density profile is obtained. This confirms that the blob electrical disconnection from the target, estimated from purely the value of $\Lambda_{\text {div }}$, does not guarantee the SOL profile flattening in TCV.

\section{Conclusion}

A set of experiments has been carried out on the TCV tokamak in order to address the role of the parallel connection length in determining SOL density profiles and its relation with filamentary dynamics. Parallel connection length has been varied by modifying the flux expansion, while keeping current and on-axis toroidal field constant. It has been observed that increasing the flux expansion slightly changes the density threshold for which the radiation front starts moving towards the X-point, without affecting the density threshold where detachment, as inferred from target integral density roll-over, is observed (cfr. Figure 4). The upstream near SOL density decay length, computed for $1 \lesssim \rho_{\Psi} \lesssim 1.03$ is found to increase strongly with the line average density, and it is largely unaffected by variation of the flux expansion. In the far SOL $\left(\rho_{\Psi} \geq 1.03\right), \lambda_{n}$ does neither depend on the line average density nor on $\mathrm{L}_{\|}$.

The observed modification of the SOL upstream density profiles with increasing density has been related to modification of filamentary blobs and divertor collisionality. We showed clearly that varying the parallel connection length at the same density increases $\Lambda_{d i v}$ without affecting blob size or upstream density profile, whereas increasing line average density causes an increase of blob size both in the near and in the far SOL. The growth of the blob size is not accompanied by an increase of the radial speed (which instead is slightly reduced) or poloidal velocity of the filaments in the far SOL. On the other hand, probe measurements suggest a reduction of the poloidal speed in the near SOL with increasing density. Fast camera measurements confirm reduction of the poloidal speed with density, although the present setup does not allow for the adjustment of the radial location of the fast camera observation. It is found that the near SOL decay length does not depend on $\Theta$ (and thus on blob size) neither on $\Lambda_{d i v}$ or $\mathrm{L}_{\|}$. However, flattening of the SOL density profile has been observed to depend on $\Theta$ and $\Lambda_{d i v}$ in the far SOL. Specifically crossing the threshold of $\Lambda_{d i v}>1$, suggested in [2] as the condition for establishment of shoulder formation, is found to be a necessary but not sufficient condition. Flat profiles are not observed for blobs in the Sheath-connected regime.

Consequently present experiments allowed establishing that increasing the parallel connection length through flux-expansion is not a viable tool to modify upstream density profiles and blob dynamics. The process of shoulder formation in TCV appears 
actually more complicated with respect to the framework proposed for AUG and JET [2], although it should be mentioned that the same framework seems less robust for the H-mode cases [8]. On the other hand, some ingredients, for example effects of neutrals which could be different in a carbon machine with a complete open divertor with respect to the AUG or JET cases, are still missing in the present description and will be investigated in forthcoming experimental campaigns.

\section{Acknowledgments}

This work has been carried out within the framework of the EUROfusion Consortium and has received funding from the Euratom research and training programme 20142018 under grant agreement No 633053. The views and opinions expressed herein do not necessarily reflect those of the European Commission 


\section{References}

[1] Myra J R, Russell D A and D'Ippolito D A 2006 Physics of Plasmas 13112502

[2] Carralero D, Manz P, Aho-Mantila L, Birkenmeier G, Brix M, Groth M, Müller H W, Stroth U, Vianello N, Wolfrum E, ASDEX Upgrade Team, JET Contributors and EUROfusion MST1 Team 2015 Phys. Rev. Let. 115215002

[3] Kočan M, Pitts R A, Arnoux G, Balboa I, de Vries P C, Dejarnac R, Furno I, Goldston R J, Gribov Y, Horaček J, Komm M, Labit B, LaBombard B, Lasnier C J, Mitteau R, Nespoli F, Pace D, Panek R, Stangeby P C, Terry J L, Tsui C and Vondracek P 2015 Nucl. Fus. 55033019

[4] LaBombard B, Boivin R L, Greenwald M, Hughes J, Lipschultz B, Mossessian D, Pitcher C S, Terry J L, Zweben S J and Group A 2001 Phys. Plasmas 82107

[5] Rudakov D L, Boedo J A, Moyer R A, Stangeby P C, Watkins J G, Whyte D G, Zeng L, Brooks N H, Doerner R P, Evans T E, Fenstermacher M E, Groth M, Hollmann E M, Krasheninnikov S I, Lasnier C J, Leonard A W, Mahdavi M A, McKee G R, McLean A G, Pigarov A Y, Wampler W R, Wang G, West W P and Wong C P C 2005 Nucl. Fus. 451589

[6] Garcia O E, Horacek J, Pitts R A, Nielsen A H, Fundamenski W, Naulin V and Rasmussen J J 2007 Nucl. Fus. 47667

[7] Carralero D, Birkenmeier G, Müller H W, Manz P, deMarne P, Müller S H, Reimold F, Stroth U, Wischmeier M, Wolfrum E and Team t A U 2014 Nucl. Fusion 54123005

[8] Carralero D, Madsen J, Artene S A, Berndt M, Birkenmeier G and Eich T 2016 Nucl. Mat. Energy

[9] Sun H J, Wolfrum E, Eich T, Kurzan B, Potzel S, Stroth U and Team t A U 2015 Plasma Physics and Controlled Fusion $\mathbf{5 7} 125011$

[10] Müller H W, Bernert M, Carralero D, Kallenbach A, Kurzan B, Scarabosio A, Sieglin B, Tophøj L, Vianello N and Wolfrum E 2015 Journ.Nucl.Mat. 463 739-743

[11] Boedo J A, Rudakov D, Moyer R, Krasheninnikov S, Whyte D, McKee G, Tynan G, Schaffer M, Stangeby P, West P, Allen S, Evans T, Fonck R, Hollmann E, Leonard A, Mahdavi A, Porter G, Tillack M and Antar G 2001 Phys. Plasmas 8 4826-4833

[12] Krasheninnikov S I 2001 Phys. Lett. A 283368

[13] Garcia O E, Bian N H, Naulin V, Nielsen A H and Rasmussen J J 2005 Physics of Plasmas 12 090701

[14] Myra J R and D'Ippolito D A 2005 Physics of Plasmas 12092511

[15] D'Ippolito D A, Myra J R and Zweben S J 2011 Physics of Plasmas 18060501

[16] Myra J R, D'Ippolito D A, Stotler D P, Zweben S J, LeBlanc B P, Menard J E, Maqueda R J and Boedo J 2006 Physics of Plasmas 13092509

[17] Easy L, Militello F, Omotani J, Dudson B, Havlíčková E, Tamain P, Naulin V and Nielsen A H 2014 Physics of Plasmas 21122515

[18] Theiler C, Furno I, Fasoli A, Ricci P, Labit B and Iraji D 2011 Physics of Plasmas 18055901

[19] Wynn A, Lipschultz B, Matthews G, Tal B, Militello F, Walkden N, Guillemaut C, Harrison J, Huber A, Kruezi U, Bric M, Nielsen A, Wiesen S, Joffrin E and Frigione D 2016 Scrape-off layer density shoulder formation and evolution in jet proceeding the 43rd Conference on Plasma Physics, Leuven, Belgium p O4.114

[20] Militello F, Garzotti L, Harrison J, Omotani J T, Scannell R, Allan S, Kirk A, Lupelli I, Thornton A J and team t M 2016 Nuclear Fusion 56016006

[21] Garcia O E, Pitts R A, Horaček J, Madsen J, Naulin V, Nielsen A H and Rasmussen J J 2007 Plasma Phys. Contr. Fus. 49 B47

[22] Coda S and TCV Team 2015 Nuclear Fusion 55104004

[23] Verhaegh K, Lipschilts B, Duval B P, Reimerdes H, Theiler C, Harrison J, Labit B, Maurizio R, Marini C, Nespoli F, Sheikh U, Tsui C, Vianello N and Vijvers W 2016 Nucl. Mat. Energy

[24] Furno I, Weisen H, Mlynar J, Pitts R A, Llobet X, Marmillod P and Pochon G P 1999 Review of Scientific Instruments $\mathbf{7 0} 4552-4556$

[25] Vijvers W A J, Canal G P, Labit B, Reimerdes H, Tal B, Coda S, De Temmerman G C, Duval 
B P, Morgan T W, Zielinski J J and Team t T 2014 Nuclear Fusion 54023009

[26] Pitts R A, Alberti S, Blanchard P, Horaček J, Reimerdes H and Stangeby P C 2003 Nuclear Fusion $431145-1166$

[27] Boedo J A, Crocker N, Chousal L, Hernandez R, Chalfant J, Kugel H, Roney P, Wertenbaker J and Team N 2009 Review of Scientific Instruments 80123506

[28] Theiler C, Furno I, Ricci P, Fasoli A, Labit B, Mueller S H and Plyushchev G 2009 Phys. Rev. Lett. 103065001

[29] Soukhanovskii V A, Bell R E, Diallo A, Gerhardt S, Kaye S, Kolemen E, LeBlanc B P, McLean A, Menard J E, Paul S F, Podestà M, Raman R, Ryutov D D, Scotti F, Kaita R, Maingi R, Mueller D M, Roquemore A L, Reimerdes H, Canal G P, Labit B, Vijvers W, Coda S, Duval B P, Morgan T, Zielinski J, De Temmerman G and Tal B 2013 Journal of Nuclear Materials 438 S96-S101

[30] Pitts R A, Duval B P, Loarte A, Moret J M, Boedo J A, Coster D, Furno I, Horaček J, Kukushkin A S, Reiter D and Rommers J 2001 Journ.Nucl.Mat. 290-293 940

[31] Theiler C, Lipschultz B, Harrison J, Labit B, Reimerdes H, Tsui C, Vijvers W A J, Boedo J A, Duval B P, Elmore S, Innocente P, Kruezi U, Lunt T, Maurizio R, Nespoli F, Sheikh U, Thornton A J, van Limpt S H M, Verhaegh K, Vianello N, Team t T and Team t E M 2017 Nuclear Fusion 57072008

[32] Stangeby P C 2000 The Plasma Boundary of Magnetic Fusion Devices Peter C. Stangeby (Taylor \& Francis)

[33] Garcia O E, Horaček J and Pitts R A 2015 Nuclear Fusion 55062002

[34] Graves J P, Horaček J, Pitts R A and Hopcraft K I 2005 Plasma Physics and Controlled Fusion 47 L1-L9

[35] Theodorsen A, Garcia O E, Horaček J, Kube R and Pitts R A 2016 Plasma Physics and Controlled Fusion $\mathbf{5 8} 044006$

[36] Walkden N R, Wynn A, Militello F, Lipschultz B, Matthews G, Guillemaut C, Harrison J, Moulton D and Contributors J 2017 Nuclear Fusion 57036016

[37] Agostini M, Terry J L, Scarin P and Zweben S J 2011 Nuclear Fusion 51053020

[38] Walkden N R, Easy L, Militello F and Omotani J T 2016 Plasma Physics and Controlled Fusion 58115010

\section{Figures}




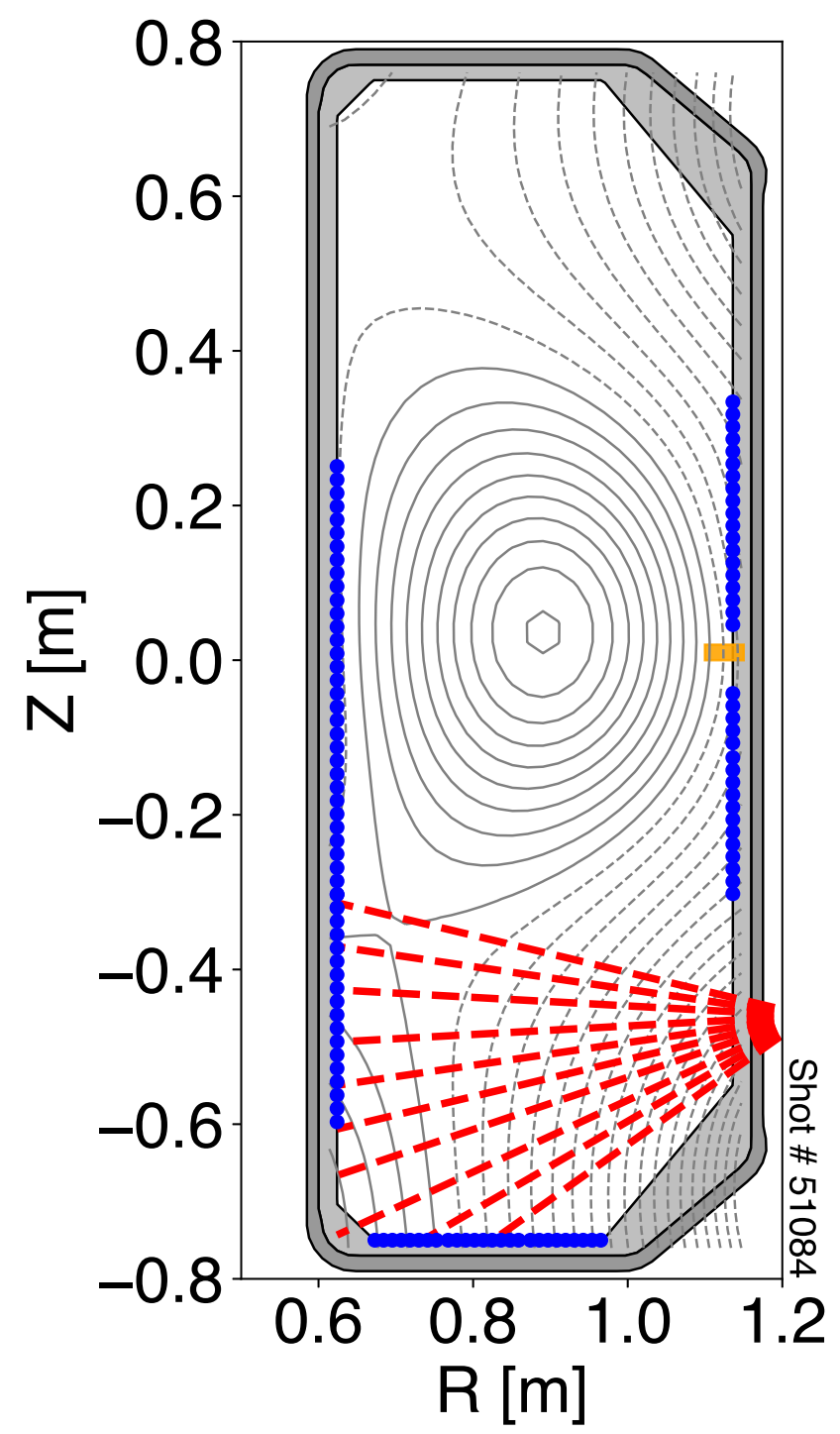

Figure 1: Poloidal cross section of the TCV tokamak and equilibrium at the smaller flux expansion explored. The blue dots indicate the position of the Langmuir probes, the red dashed line the LoS of the Bolometry. The orange box at the midplane indicates the position of fast probe reciprocation 


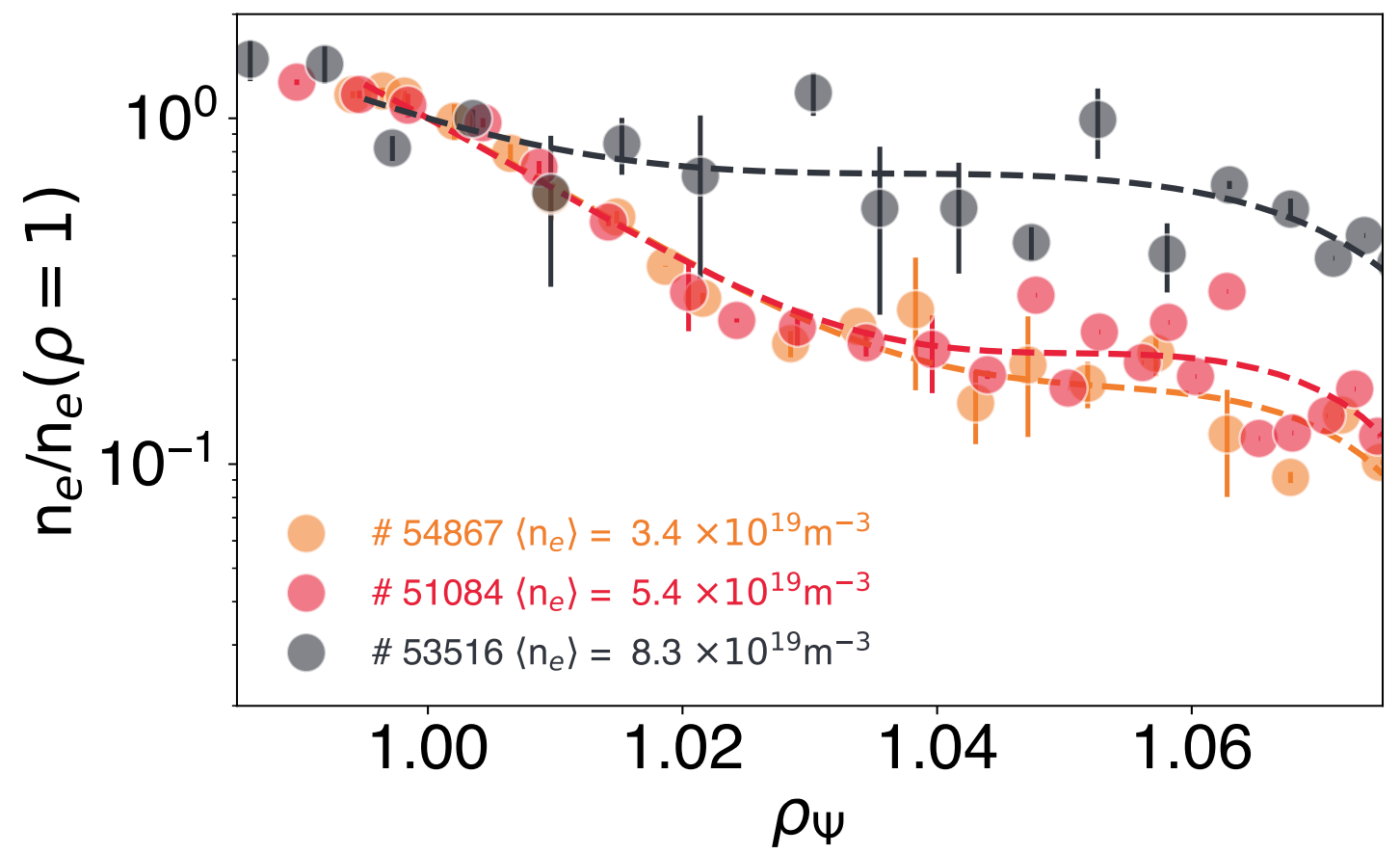

Figure 2: Upstream density profile normalized to the value at the separatrix obtained in similar L-Mode, LSN discharges at 3 values of density 

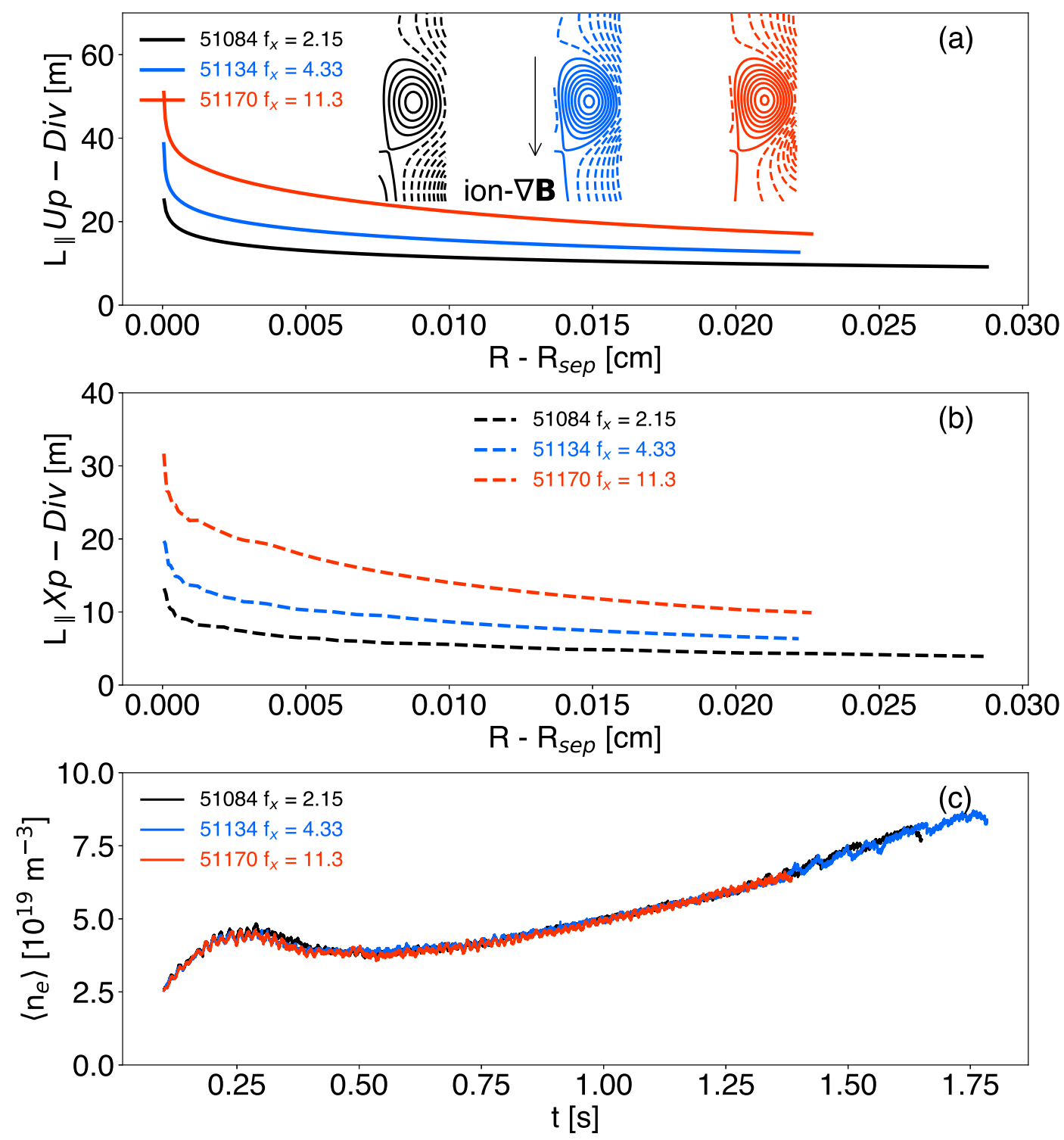

Figure 3: (a) Parallel connection length from upstream to the outer target in three different discharges with different values of flux expansin $f_{x}$. (b) Parallel connection length from X-point to the outer target (c) Line average density $\left\langle n_{e}\right\rangle$ as a function of time 



Figure 4: (a) and (b) Line integrated brightness from bolometer chords as a function of line average density for two shots at flux expansion values of respectively $f_{x} \approx 2.1$ and $f_{x} \approx 10$. (c) and (d) Spatially integrated ion flux as a function of line average density. The dashed grey line indicate the theoretically expected trend $\propto\left\langle n_{e}\right\rangle^{2}$ derived from the 2-point model 



Figure 5: (a) Upstream density profiles, normalized to the density at the separatrix at $\left\langle n_{e}\right\rangle \approx 4.5 \times 10^{19} \mathrm{~m}^{-3}$ at two values of flux expansion (2.1 and 10 respectively in black and violet) as a function of normalized poloidal flux (b) LFS target density profile at the same density as panel (a) with the same color code (c) $\Lambda_{\text {div }}$ computed with values obtained at the LFS target at the same density as panel (a) with the same color scheme. (d) Same as panel (a) at density $\left\langle n_{e}\right\rangle \approx 7 \times 10^{19} \mathrm{~m}^{-3}$ (e) Same as panel (b) at the same density as in panel (d). Same as panel (c) at the same density as in panel (d). 



Figure 6: (a) Average density decay $\lambda_{n}$ length, estimated in the near SOL $\left(1 \leq \rho_{\Psi}<\right.$ 1.025) as a function of flux expansion. The color code refers to the values of core density. (b) Same as (a) but $\lambda_{n}$ estimated between $1.025 \leq \rho_{\Psi}<1.06$. (c) Average density decay length $\lambda_{n}$ estimated in the near SOL $\left(1 \leq \rho_{\Psi}<1.025\right)$ as a function of line average density. The color code refers to values of flux expansion. (d) Same as (c) but estimated between $1.025 \leq \rho_{\Psi}<1.06$. 

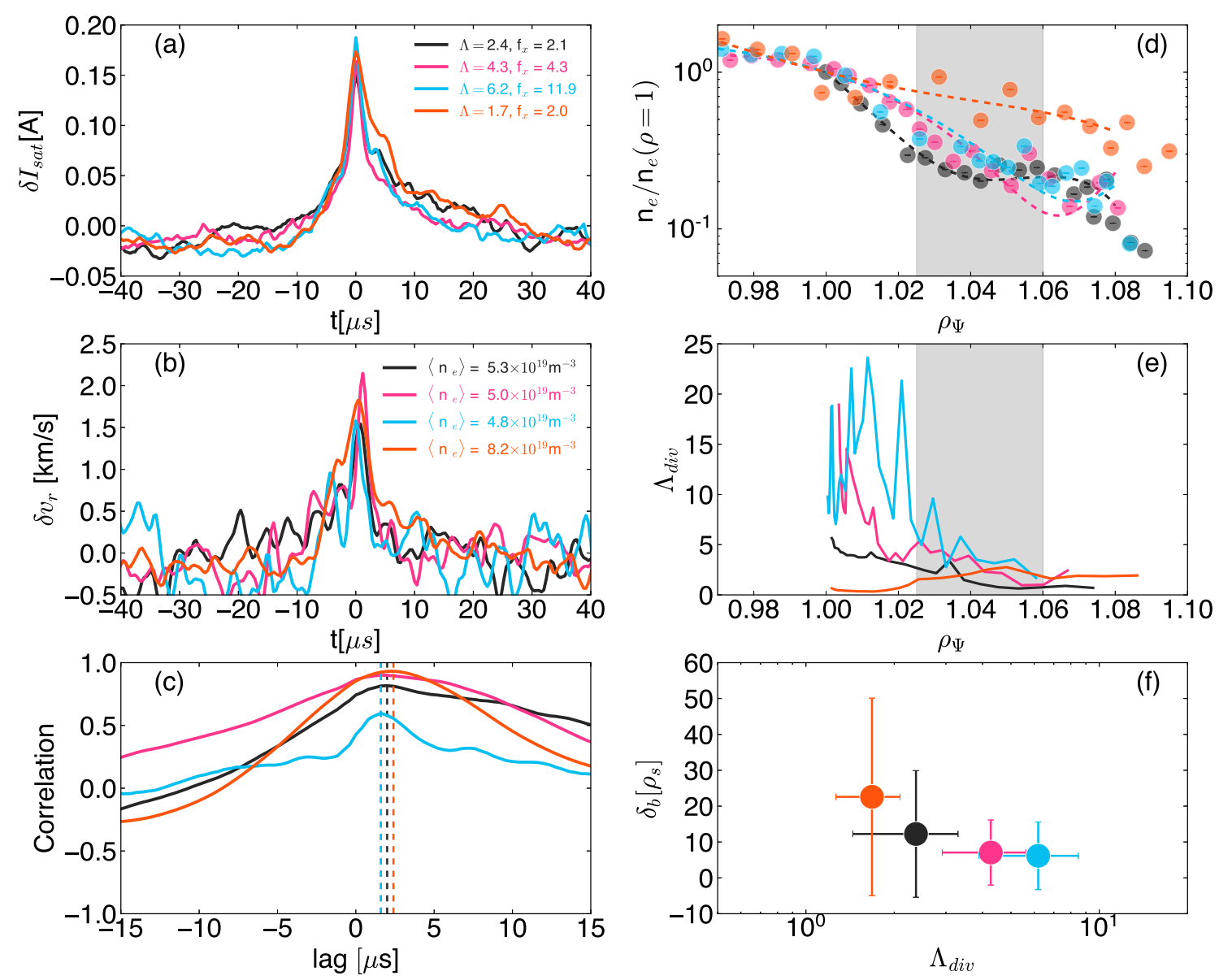

Figure 7: (a) Conditional averaged structure on ion saturation current (b) Corresponding pattern on radial $\mathbf{E} \times \mathbf{B}$ velocity (c) Cross-correlation between poloidally separated conditionally averaged sampled floating potentials. The CAS method has been applied looking to current peaks above 2.5 the standard deviation. (d) Density profiles normalized to the density at the separatrix as a function of normalized poloidal flux. (e) $\Lambda_{\text {div }}$ as a function of normalized poloidal flux (f) Blob size as a function of $\Lambda_{\text {div }}$ computed in the shaded region shown in panel (d) and (e) 

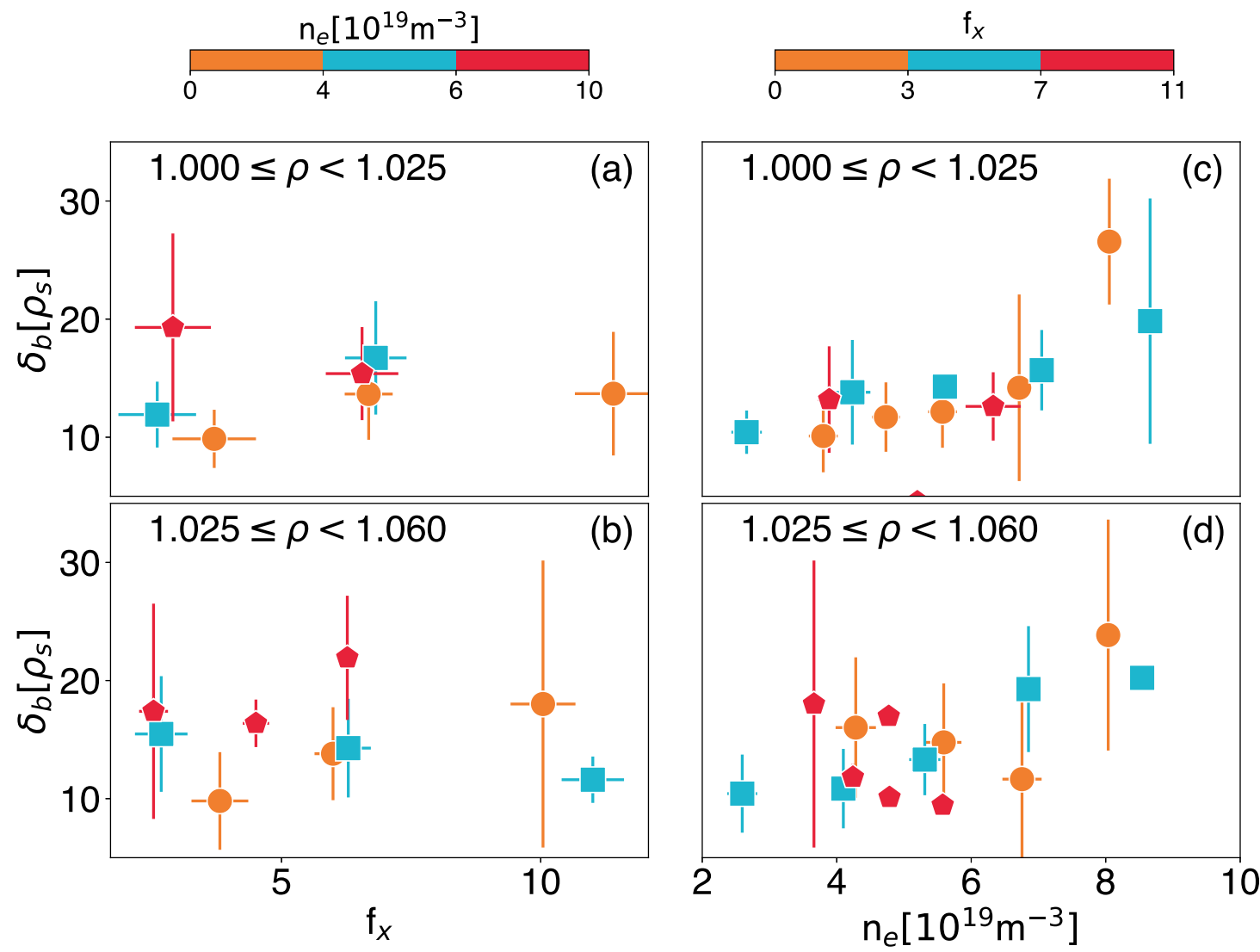

Figure 8: Blob size in $\rho_{s}$ dimension as a function of flux expansion in the near (a) and far (b) SOL. The color code refers to ranges in density. Blob size in $\rho_{s}$ dimension as a function of line average density in the near (c) and far (d) SOL. The color code refers to ranges in flux expansion. 



Figure 9: Left: FWHM of Ion saturation current structure (a), Radial velocity of filaments (b) and poloidal velocity of the filaments (c) as a function of line average density computed at radial position $1 \leq \rho_{\Psi}<1.03$. The color codes refers to classes in flux expansion. Right: same as left but computed for $1.03 \leq \rho_{\Psi}<1.06$. 

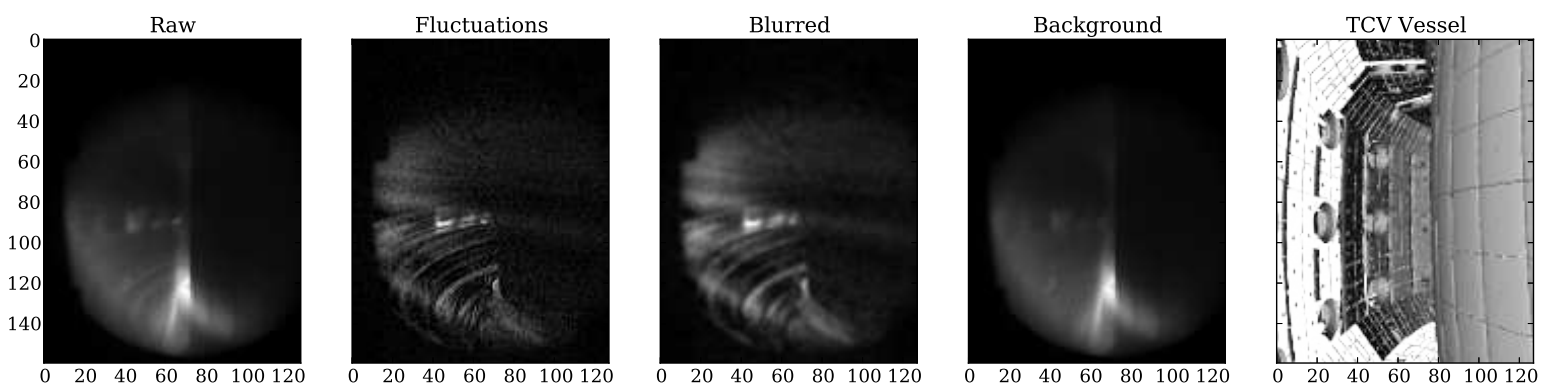

Figure 10: Image processing steps employed to extract the fluctuating component of the fast-camera movies studied here. Left: An example of raw data taken from a movie. Centre left: The fluctuating component extracted by a background subtraction. Centre right: Application of a Gaussian blur to reduce the grainy salt-noise in the image. Right: The background light emission as calculated by the background subtraction.


Figure 11: Left: Cross-correlation at zero time delay (color) and flow pattern (arrows) calculated from a 2500 frame series in shot 51134 at a density of $6 \times 10^{19} \mathrm{~m}^{-3}$. Centre and Right: The cross-correlation along a vertical line at $\mathrm{x}=85$ (blue dashed line in the left hand side figure) across a flux-expansion scan (centre) and a density scan (right). Also shown (color-coded) are the time delayed cross-correlations at $\tau=-10$ and $\tau=10$ $\mu \mathrm{s}$. 

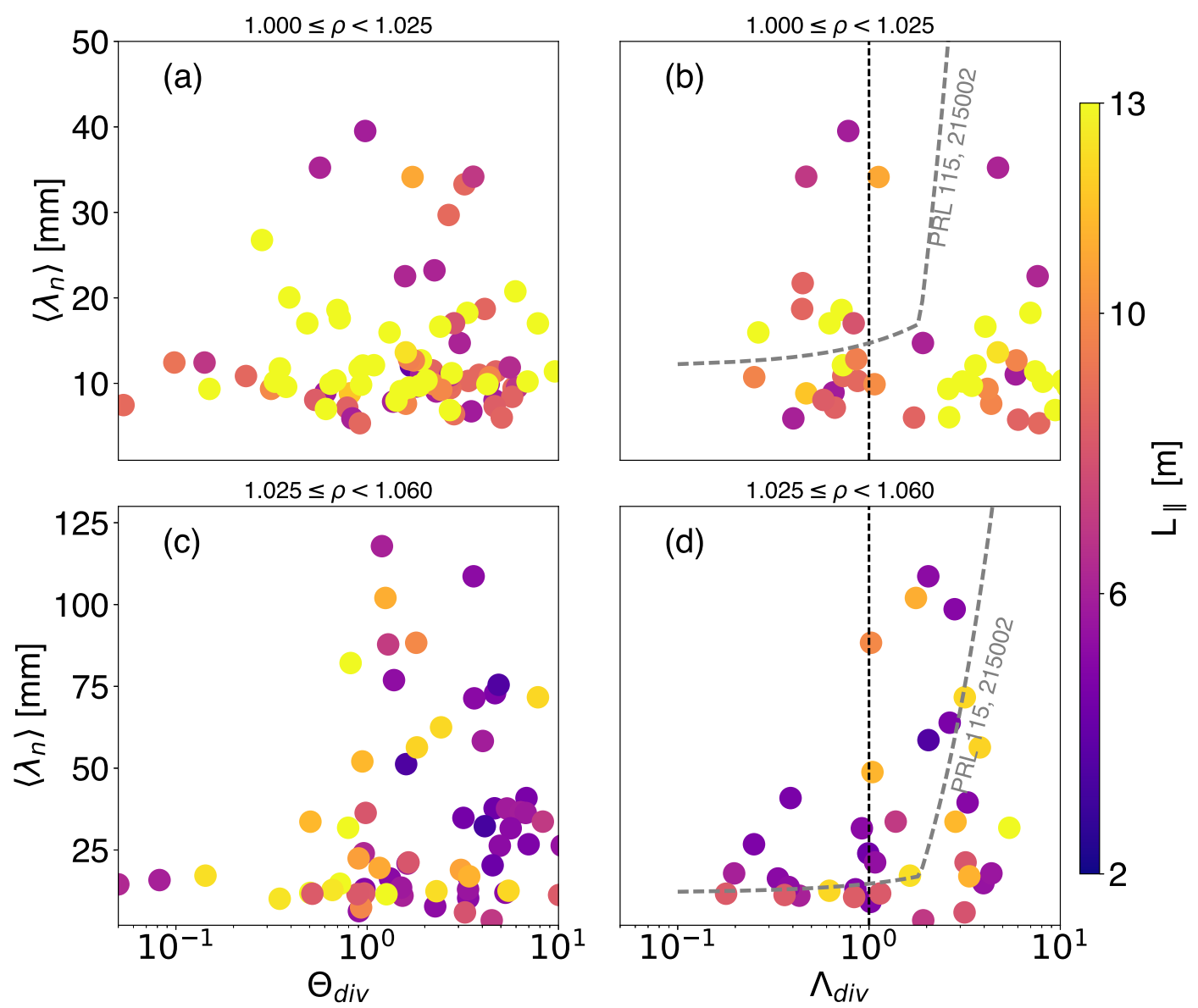

Figure 12: Density decay length as a function of $\Theta$ computed in the near (a) and far (c) SOL. Density decay length as a function of $\Lambda_{\text {div }}$ computed in the near (b) and far (d) SOL. The color is proportional to the parallel connection length. In the panel (b) and (d) we have indicated with a grey dashed line the trend observed in AUG and JET [2]. 



Figure 13: (a) $\Lambda_{\text {div }}$ vs $\Theta$ plot. The orange and blue points are those where, according to [1], the blobs should be electrically disconnected 